\title{
RESOLUTIONS
}

DE LA

\section{CONFÉRENCE INTERNATIONALE}

\author{
DE GENEVE
}

La Conférence internationale, désireuse de venir en aide aux blessés dans les cas où le service de santé militaire serait insuffisant, adopte les résolutions suivantes:

Article premier. - Il existe dans chaque pays un Comité dont le mandat consiste à concourir en temps de guerre, s'il y a lieu, par tous les moyens en son pouvoir, au service de santé des armées.

Ce Comité s'organise lui-même de la manière qui lui paraît la plus utile et la plus convenable.

ARt. 2. - Des sections, en nombre illimité, peuvent se former pour seconder ce Comité, auquel appartient la direction générale.

Art. 3. - Chaque Comité doit se mettre en rapport avec le gouvernement de son pays, pour que ses offres de service soient agréées, le cas échéant.

Art. 4. - En temps de paix, les Comités et les Sections s'occupent des moyens de se rendre véritablement utiles en temps de guerre, spécialement en préparant des secours matériels de tout genre, et en cherchant à former et à instruire des infirmiers volontaires.

ArT. 5. - En cas de guerre, les Comités des nations belligérantes fournissent, dans la mesure de leurs ressources, des secours à leurs armées respectives; en particulier ils organisent et mettent en activité les infirmiers volontaires et ils font disposer, d'accord avec l'autorité militaire, des locaux pour soigner les blessés. 
Ils peuvent solliciter le concours des Comités appartenant aux mations neutres.

ART. 6. - Sur l'appel ou avec l'agrément de l'autorité militaire les Comités envoient des infirmiers volontaires sur le champ de bataille. Ils les mettent alors sous la direction des chefs militaires.

Art. 7. - Les infirmiers volontaires employés à la suite des armées doivent être pourvus, par leurs Comités respectifs, de tout ce qui est nécessaire à leur entretien.

Art. 8. - Ils portent dans tous les pays, comme signe distinctif uniforme, un brassard blanc avec une croix rouge.

Art. 9. - Les Comités et les Sections des divers pays peuvent se réunir en Congrès internationaux, pour se communiquer leurs expériences et se concerter sur les mesures à prendre dans l'intérêt de l'œuvre.

Art. 10. - L'échange des communications entre les Comités des diverses nations, se fait provisoirement par l'entremise du Comité de Genève.

Indépendamment des résolutions ci-dessus, la Conférence émet les vceux suivants :

a. Que les gouvernements accordent leur haute protection aux Comités de secours qui se formeront, et facilitent autant que possible l'accomplissement de leur mandat.

b. Que la neutralisation soit proclamée, en temps de guerre, par les nations belligérantes pour les ambulances et les hòpitaux, et qu'elle soit égálement admise, de la manière la plus complète, pour le personnel sanitaire officiel, pour les infirmiers volontaires, pour les habitants du pays qui iront secourir les blessés, et pour les blessés eux-mêmes. 
c. Qu'un signe distinctif identique soit admis pour les colps sanitaires de toutes les armées, ou tout au moins pour les personnes d'une même armée attachées à ce service.

Qu'un drapeau identique soit aussi adopté, dans lous les pays, pour les ambulances et les hôpitaux.

$$
\begin{aligned}
& \text { Le secrélaire de la Conférence. } \\
& \text { J.-Henry DUNANT. }
\end{aligned}
$$

Genève, octobre 1863. 